\title{
A NEW METHOD BASED ON IMMUNE ALGORITHM TO SOLVE THE UNIT COMMITMENT PROBLEM
}

\author{
Wei Li, Deren Sheng, Jianhong Chen, Zhenfu Yuan, Kefa Cen \\ College of Mechanical and Energy Engineering, Zhejiang University, 310027 China, \\ Email:energy@cmee.zju.edu.cn
}

\begin{abstract}
A new method based on immune algorithm to solve the thermal unit commitment problem(UCP) is proposed. Through analysis of mathematical model of UCP, application method of immune algorithm was discussed in detail. In the algorithm, the objective function was deemed antigen and the solutions were deemed antibodies. If an antibody fits the antigen best, this antibody was deemed the optimum solution. Encoding the continuous operating time shortened the code length and the searching speed of algorithm was improved greatly. The proposed method was applied in a ten-unit system for a 24 hours period. The calculation results showed immune algorithm had well global searching performance and it was efficient to use this algorithm to solve UCP.
\end{abstract}

Key words: Unit Commitment Problem(UCP), Immune Algorithm, Optimization, Thermal Unit

\section{INTRODUCTION}

The thermal unit commitment problem (UCP) is a non-linear combinatorial optimization problem. The task of UCP is to find optimum units combination to meet the requirements of the power system. According to references, the efficiency can be improved by $1 \%$ to $2.5 \%$ through optimization of unit commitment according to scheduling experience(Pei et al., 2001). So researchers try to solve the unit commitment problem using all kinds of intelligent algorithms for long time.

Please use the following format when citing this chapter:

Li, Wei, Sheng, Deren, Chen, Jianhong, Yuan, Zhenfu, Cen, Kefa, 2006, in International Federation for Information Processing (IFIP), Volume 207, Knowledge Enterprise: Intelligent Strategies In Product Design, Manufacturing, and Management, eds. K. Wang, Kovacs G., Wozny M., Fang M., (Boston: Springer), pp. 840-846. 
There are several conventional methods to solve the unit commitment problem, such as priority list, dynamic programming, intege programming, linear programming, Lagrangian relaxation method and so on. Because of the complexity of UCP and the numerous constraints, the optimum solution can't be achieved efficiently using these methods (Cai et al., 1997). Recently genetic algorithm is applied to tackle UCP. However genetic algorithm is easy to suffer from local optimum solution for its poor diversity and premature convergence. A new method based on immune algorithm is proposed to handle UCP. Immune algorithm is a global search method and it has memorization capabilities and good diversity. So this algorithm has good convergence and can search global optimum solution effectively.

\section{UCP FORMULATIONS}

1. Objective functions (Cai et al., 1997)

The task of unit commitment is to minimize the total fuel consumption of the generating units including operation consumption and start-up consumption. The objective functions can be expressed as below:

$$
\min F\left(U_{i t}, P_{i t}\right)=\sum_{i=1}^{24} \sum_{i=1}^{G}\left[U_{i t} F_{i}\left(P_{i t}\right)+U_{i t}\left(1-U_{i(t-1)}\right) S_{i}\right]
$$

where $F\left(U_{i t}, P_{i t}\right)$ is the total fuel consumption function, $U_{i t}$ is the on/off status of unit $i$ at hour $t, U_{i t}=1$ if unit $\mathrm{i}$ is on and $U_{i t}=0$ if unit i is off, $P_{i t}$ is the generation output of unit $i$ at hour $t, F_{i}\left(P_{i t}\right)$ is the fuel consumption of unit $i$ at hour $\mathrm{t}, \mathrm{G}$ is the total number of units, $S_{i}$ is the start-up cost of unit i.

2. Constraints

Six constraints should be satisfied. There are electric power balance equation, output limits of generating units, spinning reserve considerations, limits of ramp rates, start-up and down frequency, minimum up-time and minimum down-time.

\section{INTRODUCTION OF IMMUNE ALGORITHM}

\subsection{Summary of immune algorithm}

The immune algorithm is inspired by the human immune system that protects our bodies from attack of foreign substance (called antigen). In the 
algorithm, the objective functions and constraints were deemed antigens. The feasible solutions were deemed antibodies. A computation of affinity was embedded for determining the promotion or suppression of antibodies production. Therefore the diversity of antibodies were kept by the boost or restriction of antibody generations. If an antibody has maximum affinity, this antibody is deemed the optimum solution to the optimization problem.

Immune algorithm has some distinct characteristics as follows:

- It operates on the feasible solutions that guarantee good convergence.

- The diversity is kept by boost or restriction of antibody generations.

\subsection{Computation of immune algorithm}

1) Computation of affinity (Gao, 2001)

If the antibody pool is composed of $N$ antibodies, each of which has $M$ genes. The entropy $H_{j}(N)$ of the jth gene can be expressed as follows:

$$
H_{j}(N)=\sum_{i=1}^{N}-p_{i j} \log p_{i j}
$$

where $p_{i j}$ is the probability that the ith allele comes out of the jth gene.

Then the average information entropy can be expressed as below:

$$
H(N)=\left(\sum_{j=1}^{M} H_{j}(N)\right) / M
$$

The affinity between antibodies can evaluate the diversity of antibodies. If antibodies are more similar, the affinity between antibodies is higher. The computing formulation can be represented as below:

$$
\alpha_{v, w}=1 /(1+H(2))
$$

where $H(2)$ is the information entropy between antibody $\mathrm{v}$ and $\mathrm{w}$.

2) Computation of antibody concentration

The antibody concentration is the proportion of some similar antibodies to the whole population. It can be expressed as follows:

$$
c_{v}=\frac{\sum_{i=1}^{N} S_{v, w}}{N}, S_{v, w}= \begin{cases}1, & \text { 当 } \alpha_{v, w} \geq T_{a c 1} \\ 0, & \text { 当 } \alpha_{v, w}<T_{a c 1}\end{cases}
$$


where $\mathrm{N}$ is the total number of antibody population, $\mathrm{T}_{\mathrm{acl}}$ is the threshold of antibody concentration.

3) Computation of antibody viability

$$
e_{v}=A_{v} \prod_{s=1}^{S} \frac{1-L_{v, s}^{k}}{c_{v} \sum_{i=1}^{N} A_{i}}, L_{v, s}= \begin{cases}\alpha_{v, s} & \alpha_{v, s} \geq \mathrm{T}_{a c 2} \\ 0, & \alpha_{v, s}<T_{a c 2}\end{cases}
$$

where $A_{i}$ is the sufficiency value of antibody, $\mathrm{S}$ is the total number of the suppressor cells, $\mathrm{k}$ is the suppressor index, $\mathrm{T}_{\mathrm{ac} 2}$ is the viability threshold.

4) Computation process of immune algorithm

Step 1: Initialization.

Some parameters of immune algorithm are set, such as the population size, the total number of memory cells, the concentration threshold, crossover rate, mutation rate and so on.

Step 2: Antigen recognition.

In the immune algorithm, the objective function of unit commitment problem and constraints are deemed as antigen.

Step 3: Generation of initial antibody.

Initial antibody generates according to the encoding method based on binary code. A group of genes form an antibody. Each antibody represents a feasible solution to the optimization problem.

Step 4: Computation of affinity.

Step 5: Computation of antibodies concentration.

Step 6: Differentiation of memory cells.

If some antibody's concentration is over threshold, the memory cell generates to memorize the local optimum solution.

Step 7: Boost or restriction of antibodies.

The antibody with low concentration will most possibly survive to next generation, whereas the suppressor cells will restrict the antibodies with high concentration. Thus the diversity of antibody is kept.

Step 8: Generation of new antibodies.

The new antibodies generate after crossover operation, mutation operation and immune complementarity. Immune complementarity means that the antibody has low viability will be replaced with a new antibody generates randomly.

Step 9: Termination judgment.

The calculation will stop if the termination condition is satisfied. Otherwise the calculation will execute from step 4 to step 8 .

Step 10: Output optimum solution. 


\section{IMPLEMENTATION OF IMMUNE ALGORITHM}

\subsection{Encoding method of antibody}

If the operating status of one unit which in single time-interval is represented by one-bit binary code, for $G$ units and 24 hours, the length of an antibody is $\mathrm{L}=24^{*} \mathrm{G}$ bits. Since the code length is long, the code string that has better mode is easily destroyed under crossover and mutation operation. Considering the constraint of daily start-up and down frequency, some researchers proposed to encode the variable position of units operating status. There are 24 intervals per day and 23 variable positions altogether. One variable position can be represented by 5-bits binary code. It only needs 15 bits binary code if the maximum start-up and down frequency is three.

In addition, generating units should also meet the constraint of minimum up-time and minimum down-time. A new encoding method is proposed, viz. to encode the continuous operating time of each unit. For the maximum continuous operating time is 24 , it can be represented by a 5-bits binary code. If the maximum start-up and down frequency is three, then there are four status continuous time altogether. We only need to encode the former three continuous times. Suppose the initial status is on, the encoding method of antibody is given as below:

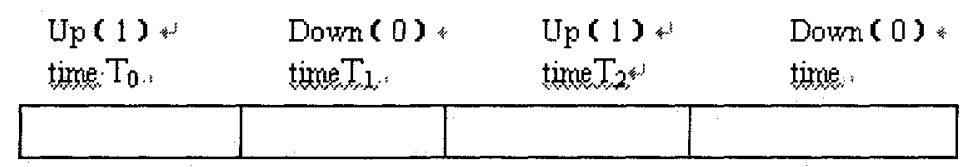

Figure l.encoding figure

In this way, 15-bits binary code is needed to express operating status for 24 hours of each unit. For example, assuming the initial status of one unit is 1 , antibody code is 001010011100110 , that denotes the operating status in the 1 st5 th hour for this unit is 1 , in the 6 th-12th hour is 0 , in the 13 th-18th hour is 1 , in the 19th-24th hour is 0 . Encoding this way not only shortens the code length, but also satisfies the constraint of the start-up and down frequency and the constraint of the minimum continuous operating time so as to quicken the searching speed of immune algorithm.

\subsection{An example}

The proposed method was tested on a system consist of ten units. Their characteristic data and the data of load are listed in Ref. Han et al., 1994. 
And tab.2 tabulated the optimum unit commitment for a $24 \mathrm{~h}$ period. In immune algorithm, the population size is 100 , crossover rate is 0.75 , mutation rate is 0.05 , the concentration threshold is 0.85 , the viability threshold is 0.8 , and selection rate is 0.8 .

The calculation result using immune algorithm was compared with that using other optimization methods in tab.1. The computing total fuel consumption using the proposed method was 78970.3 tons coals. It was $0.48 \%$ better than the calculation result using dynamic programming method, and less than the calculation result using modified genetic algorithm by 27.7 tons. From calculation process, the optimum solution could be achieved through less than twenty generations. The calculation result showed that this method had good convergence and global searching performance and it is effective and efficient to solve the unit commitment problem.

Table 1 calculation results using different optimization methods

\begin{tabular}{cc}
\hline Optimization methods & $\begin{array}{c}\text { Total fuel } \\
\text { consumption (tons) }\end{array}$ \\
\hline Lagrangian relaxation & 80766 \\
Heuristic genetic algorithm & 79807 \\
Dynamic programming & 79349 \\
Genetic algorithm in ref.Shi et al., 2002 & 79004 \\
Improved genetic algorithm in ref.Han et al., 1994 & 81245.4 \\
Modified genetic algorithm in ref.Pei et al., 2001 & 78998 \\
Immune algorithm & 78970.3 \\
\hline
\end{tabular}

\section{CONCLUSIONS}

As a novel optimization method, immune algorithm has some distinct features, such as good diversity, boost or restriction mechanism between antibodies and global searching ability and so on. Using immune algorithm to optimize unit commitment can achieve the optimum solution efficiently. The calculation results showed that it is promising to use immune algorithm to solve UCP and other optimization problem in thermal power plant.

\section{REFERENCES}

1. Cai Chao-hao, Cai Yuan-yu. 1997. Optimization of unit commitment by genetic algorithm. Power System Technology, 21(1):44-47,51.(in Chinese)

2. Gao Jie. 2001. the Application of the immune algorithm for power network planning. Systems Engineering-Theory \& Practice, 5:119-123.(in Chinese) 
3. Han Xue-shan, Liu Zhuo. 1994. Optimal unit commitment considering unit's ramp-rate limits. Power System Technology, 6:11-16.(in Chinese)

4. Sun Yongzhi, Wei Wei. 2002. Solution of optimal power flow problem based on artificial immune algorithm. Automation of Electric Power Systems, 26(12):30-34. (in Chinese)

5. Pei Jin-yong, Lai Yi-fei, Chen Pin et al. 2001.Modified genetic algorithm for optimizing combination of units. Engineering Journal of Wuhan University, 34(1):72-76.(in Chinese)

6. Shi Qi, Li Cheng-jun, Wang Jin-wen. 2002. the Application of genetic algorithm to shortterm generation scheduling. Proceedings of the EPSA, 14(2):56-59. (in Chinese)

7. Shyh-Jier Huang. 1999. Enhancement of thermal unit commitment using immune algorithms based optimization approaches. Electrical Power and Energy Systems, 21:245252.

8. S.O. Orero, M.R.Irving. 1997. A combination of the genetic algorithm and Lagrangian relaxation decomposition techniques for the generation unit commitment problem. Electric Power Systems Research, 43:149-156. 